\title{
METRIZABILITY OF ADJUNCTION SPACES
}

CARLOS J. R. BORGES ${ }^{1}$

In a recent conversation with E. A. Michael and D. Hyman the following natural question was raised: Are the $M$-spaces of Hyman (see Definition 3.1) metrizable whenever they are first countable? We will answer this question affirmatively. Indeed, we will prove the somewhat stronger result that the $M$-spaces of Hyman are metrizable whenever they are of pointwise countable type (see Definition 1.1 and ensuing paragraph).

It turns out that to answer the preceding question the major difficulty involves the metrizability of adjunction spaces. All our spaces are assumed to be $T_{1}$.

\section{Preliminary analysis.}

Definition 1.1 (ARHANGEL'SkIIי). A topological space $X$ is said to be of pointwise countable type if each point $p \in X$ is contained in a compact subset $C_{p}$ of $X$ of countable character (i.e. $C_{p}$ has a countable neighborhood base in $X$ ).

Clearly all first countable spaces are of pointwise countable type. Furthermore, the $p$-spaces of Arhangel'skir (hence, the topologically complete spaces in the sense of Čech) are also of pointwise countable type (see Theorem 9 and the corollary to Theorem 8 in [1]). Finally, Arhangel'skii has proved that, in the presence of paracompactness, the $r$-spaces of Michael [7] are equivalent to the $q$-spaces of Michael [6] as well as to the spaces of pointwise countable type (see the first paragraph of $\S 3$ of [7]).

Our work in $\S \S 2$ and 3 will depend heavily on the following result, which is essentially due to Arhangel'skiǐ [2].

Proposition 1.2. Let $X$ be a completely regular space of pointwise countable type. If every compact subset of $X$ is first countable then $X$ is first countable.

Proof. Essentially contained in the proof of Theorem 3.11 of [2] (note that, in Arhangel'skiir's proof, Proposition 3.3 should be quoted instead of Proposition 3.2).

Definition 1.3. For any spaces $X$ and $Y$, a closed subset $A$ of $X$ and a continuous function $f: A \rightarrow Y$, we call the tuple $(X, A, f, Y)$ an

Received by the editors May 16, 1969.

1 This research was supported by the NSF grant GP-6390. 
adjunction tuple and the quotient space $X \cup_{f} Y$, obtained from (the disjoint topological union) $X \vee Y$ by identifying each $x \in A$ with $f(x)$, the adjunction space. We denote the natural map from $X \vee Y$ onto $X \cup, Y$ by $p$. (Note that $p \mid(X-A)$ and $p \mid Y$ are homeomorphisms; thus we will of ten identify $Y$ with $p(Y)$, whenever convenient.)

2. Main result. Throughout this section let $X$ and $Y$ be metrizable spaces, $A$ a closed subset of $X$ and $f: A \rightarrow Y$ a continuous function such that $X \cup_{f} Y$ is a space of pointwise countable type.

Our main result (i.e. Theorem 2.6) has a surprisingly short proof (at least, when compared with our original proof) thanks to Lemma 2.2, which is due to Professor E. Michael.

Lemma 2.1. The map $p: X \vee Y \rightarrow X \cup_{f} Y$ is pseudo-open (i.e. if $V$ is a neighborhood of $p^{-1}(w)$ then $p(V)$ is a neighborhood of $\left.w\right)$.

Proof. We will first show that $X \cup_{f} Y$ is first countable: By Theorem 6.2 and Corollary 8.3 of [3], each compact subset of $X \cup_{f} Y$ is metrizable and hence first countable. Therefore $X$ is first countable, by our Proposition 1.2.

The remainder of the proof is essentially the same as the proof of Lemma 1 of Stone [12].

Lemma 2.2. If $B \subset(X-A)^{-}$is closed in $X$, then $f(B)$ is closed in $Y$.

Let $y \in Y-f(B)$. Then $B$ and $f^{-1}(y)$ are disjoint closed subsets of $X$, and thus there are disjoint open sets $U, V$ in $X$ with $B \subset U$ and $f^{-1}(y) \subset V$. Let $W=Y \cup V$. Then $W$ is a neighborhood of $p^{-1} p(y)$ $=\{y\} \cup f^{-1}(y)$ in $X \vee Y$, so $p(W)$ is a neighborhood of $p(y)$ in $X \cup, Y$, by Lemma 2.1. Let $G$ be the interior of $p(W)$. Then $p^{-1}(G)$ does not intersect $U-A$, hence not $B$, and hence not $f(B)$. Thus $y$ is not in the closure of $f(B)$ in $Y$.

Lemma 2.3. The equalities

$$
\begin{aligned}
& (p(X-A))^{-}=p(X-A) \cup\left\{y \in Y \mid f^{-1}(y) \cap \text { bdry } A \neq \varnothing\right\}, \\
& p\left((X-A)^{-}\right)=(p(X-A))^{-}
\end{aligned}
$$

are valid.

Proof. The first equality is easily seen because of Lemma 2.1 and the fact that $p^{-1} p(X-A)=X-A$, and the second equality follows from the first and the continuity of $p$.

Lemma 2.4. The map $q=p \mid(X-A)-$ is a closed map from $(X-A)^{-}$ onto $(p(X-A))^{-}$. 
Proof. Because of Lemma 2.3 we only need prove that $q$ is closed. However, $q$ is obviously closed because of Lemma 2.2 and the fact that $q \mid(X-A)$ is a homeomorphism.

Lemma 2.5. The space $p\left((X-A)^{-}\right)$is metrizable.

Proof. Using Theorem 2.1 and Corollary 2.2 of [6] and our Lemma 2.4 , one easily proves that the boundary of $q^{-1}(y)$ is compact for each $y \in p(X-A)^{-}$. Therefore $p\left((X-A)^{-}\right)$is metrizable, by Theorem 1 of Stone [12] and our Lemma 2.4.

We are now ready for our main result, which is analogous to Proposition 3.1 of [7]:

THEOREM 2.6. If the space $X \cup_{f} Y$ is a space of pointwise countable type, and $X$ and $Y$ are metrizable then $X \cup_{f} Y$ is metrizable.

Proof. By Lemmas 2.5 and 2.3, we get that

$$
X \cup_{f} Y=Y \cup p\left((X-A)^{-}\right),
$$

with $Y$ and $p\left((X-A)^{-}\right)$closed metrizable subspaces of $X \cup_{f} Y$. Therefore, the space $X \cup_{f} Y$ is metrizable by Theorem 2 of Nagata [9].

3. Metrizability of paracomplex spaces. We will now settle the question of the metrizability of Hyman's $M$-spaces. We start with their definition and some pertinent comments.

Definition 3.1 (Hyman's $M$-spaces-SeE Definition 3.3 and Theorem 3.4 of Hyman [5]). An $M_{0}$-space is a metric space. Assuming that $M_{n}$-spaces have been defined, define a space $Z$ to be an $M_{n+1^{-}}$ space if there exists an adjunction tuple $(X, A, f, Y)$ such that $X$ is an $M_{0}$-space, $Y$ is an $M_{n}$-space and $Z$ is homeomorphic to $X \cup_{f} Y$. A space $T$ is said to be an $M_{\infty}$-space if $T$ is an $M_{n}$-space for some $n \geqq 0$. Finally a space $S$ is said to be an $M$-space if there exists a sequence $\left\{S_{n}\right\}_{n=1}^{\infty}$ of closed $M_{\infty}$-subspaces of $S$ such that $S$ is the weak union of the spaces $S_{n}$ for $n=1,2, \ldots$ (i.e. $S=\cup_{n=1}^{\infty} S_{n}, S_{n} \subset S_{n+1}$ for each $n$, and a subset $A$ of $S$ is closed if and only if $A \cap S_{n}$ is a closed subset of $S_{n}$ for each $n$ ).

Hyman's terminology for this new class of spaces seems quite undesirable since we already have the $M$-spaces of Morita [8] and the $M_{i}$-spaces $(i=1,2,3)$ of Ceder [4]. Since Hyman's $M$-spaces are an obvious generalization of the $\mathrm{CW}$-complexes of Whitehead (as pointed out by Hyman) we propose that Hyman's $M$-spaces be called PARACOMPLEX and that his $M_{n}$-spaces be called $n$-PARACOMPLEX, a terminology which we will use from now on. 
The class of paracomplex spaces has many remarkable properties, but the one that fascinates us the most is the following result of Hyman (see Theorem 4.6 of [5]):

Proposition 3.2. The class of paracomplex spaces is the smallest class of topological spaces which contains the class of metric spaces and is closed under adjunctions and weak unions.

A close examination of Definition 3.1 reveals that before one can prove that paracomplex spaces of pointwise countable type are metrizable one must prove the following statements:

(A) If a space $X$ of pointwise countable type is the weak union of metrizable spaces then $X$ is metrizable.

(B) If $X \cup \cup_{f} Y$ is a space of pointwise countable type, and $X$ and $Y$ are metrizable then $X \cup_{f} Y$ is metrizable.

Since we have already proved (B) in $\S 2$, we will now prove (A). Indeed we will prove a much stronger version of (A) (i.e. Theorem 3.6).

Definition 3.3 (E. Michael). Let $X$ be a topological space, and $B$ a collection of closed subsets of $X$. Then $B$ dominates $X$ if, whenever $A \subset X$ has a closed intersection with every element of some subcollection $B_{1}$ of $B$ which covers $A$, then $A$ is closed.

Lemma 3.4. If a space $X$ is the weak union of the subspaces $X_{n}$ for $n=1,2, \cdots$, then $X$ is dominated by $\left\{X_{n}\right\}_{n=1}^{\infty}$.

Proof. Trivial, since $X_{n} \subset X_{n+1}$ for each $n$.

Lemma 3.5. If a space $X$ is dominated by a collection $x$ of subspaces then every compact subset of $X$ is contained in finitely many elements of $x$.

Proof. Essentially the same as the proof of Lemma 9.3 of [11].

TheOREm 3.6. Let $X$ be a space of pointwise countable type. If $X$ is dominated by a collection $X$ of metrizable suspaces then $X$ is metrizable.

Proof. By Theorem 7.2 of [1], $X$ is stratifiable and hence paracompact (this also follows from results of $\mathrm{E}$. Michael and K. Morita). Consequently we only need show that $X$ is locally metrizable to complete the proof, because of Theorem 3 of Smirnov [10].

We will first show that $X$ is first countable: By our Lemma 3.5 and Theorem 2 of [9], each compact subset of $X$ is metrizable and thus first countable. Therefore $X$ is first countable, by Proposition 1.2.

Now let $p \in X$ and let $\left\{U_{n}\right\}_{n-1}$ be a neighborhood base for $p$. We 
will show that, for some $n, U_{n}$ is covered by finitely many elements of $X$ : Suppose not. Then, by induction, one can easily find $X_{i} \in X$ and $p_{i} \in X-\{p\}$ for $i=1,2, \cdots$, satisfying

(a) $p_{i} \in U_{i} \cap X_{i}$

(b) $P_{k} \notin X_{i}$ for $i<k$.

Then the set $A=\left\{p_{1}, p_{2}, \cdots\right\}$ is a closed subset of $X$, since each $A \cap X_{i}$ is a finite (and hence closed) subset of $X_{i}$ and $A \subset \cup_{i=1}^{\infty} X_{i}$. Therefore $X-A$ is an open subset of $X$ which contains $p$ but contains no $U_{n}$, a contradiction.

We have thus proved that each $p \in X$ has a neighborhood which is covered by finitely many closed metrizable subspaces, which implies that $X$ is locally metrizable, because of Theorem 2 of [9]. This completes the proof.

COROLlary 3.7. If a space of pointwise countable type is the weak union of metrizable subspaces $X_{n}$ for $n=1,2, \cdots$, then $X$ is metrizable.

Proof. Immediate, by Lemma 3.4.

THEOREM 3.8. If $X$ is an n-paracomplex space of pointwise countable type, for some $n \geqq 0$, then $X$ is metrizable.

Proof. Certainly $X$ is metrizable if $n=0$, by Definition 3.1. By induction, suppose that any $k$-paracomplex space is metrizable and let us show that any $(k+1)$-paracomplex space $Z$ of pointwise countable type is metrizable. But $Z$ is (homeomorphic to) an adjunction space $X \cup_{f} Y$, with $X$ and $Y$ metrizable spaces (note that $Y$ is a closed $k$-paracomplex subspace of $Z$; therefore, $Y$ is of pointwise countable type and thus metrizable, by the induction hypothesis), and thus $Z$ is metrizable, because of Theorem 2.6. This completes the proof.

Theorem 3.9. A paracomplex space is metrizable if and only if it is a space of pointwise countable type.

Proof. Immediate from Theorem 3.8 and Corollary 3.7.

\section{REFERENCES}

1. A. Arhangel'ski1, On a class of spaces containing all metric and all locally bicompact spaces, Dokl. Akad. Nauk SSSR 151 (1963), 751-754=Soviet Math. Dokl. 4 (1963), 1051-1055. MR 27 \#2959.

2. - Bicompact sets and the topology of spaces, Trudy Moskov. Mat. Obšc. 13 (1965), 3-55 = Trans. Moscow Math. Soc. 1965, 1-62. MR 33 \#3251.

3. C. J. R. Borges, On stratifiable spaces, Pacific J. Math. 17 (1966), 1-16, MR 32 \#6409.

4. J. G. Ceder, Some generalizations of metric spaces, Pacific J. Math. 11 (1961), 105-125. MR 24 A \#1707. 
5. D. M. Hyman, A category slightly larger than the metric and CW-categories, Michigan Math. J. 15 (1968), 193-214. MR 37 \#4772.

6. E. A. Michael, $A$ note on closed maps and compact sets, Israel J. Math. 2 (1964), 173-176. MR 31 \#1659.

7. - No-spaces, J. Math. Mech. 15 (1966), 983-1002. MR 34 \#6723.

8. K. Morita, On the product of a normal space with a metric space, Proc. Japan Acad. 39 (1963), 148-150. MR 27 \#721.

9. J. Nagata, On a necessary and sufficient condition of metrizability, J. Inst. Polytech. Osaka City Univ. Ser. A Math. 1 (1950), 93-100. MR 13, 264.

10. Ju. M. Smirnov, On the metrization of topological spaces, Uspehi Mat. Nauk 6 (1951), no. 6 (46), 100-111; English transl., Amer. Math. Soc. Transl. (1) 8 (1962), 6277. MR 14, 70.

11. N. E. Steenrod, A convenient category of topological spaces, Michigan Math. J. 14 (1967), 133-152. MR 35 \#970.

12. A. H. Stone, Metrizability of decomposition spaces, Proc. Amer. Math. Soc. 7 (1956), 690-700. MR 19, 299.

University of California, Davis 